\title{
Myelodysplastic Syndromes with Bone Marrow Fibrosis: An Update
}

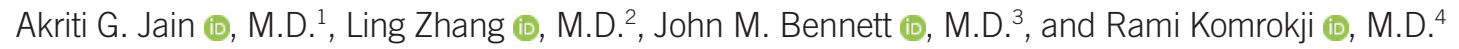 \\ Departments of ${ }^{1} \mathrm{Hematology}$ and Oncology and ${ }^{2} \mathrm{Hematopathology} \mathrm{and} \mathrm{Laboratory} \mathrm{Medicine,} \mathrm{H.} \mathrm{Lee} \mathrm{Moffitt} \mathrm{Cancer} \mathrm{Center} \mathrm{and} \mathrm{Research} \mathrm{Institute,} \mathrm{Tampa,}$ \\ FL, USA; ${ }^{3}$ Hematopathology Division, Department of Pathology, University of Rochester Medical Center, Rochester, NY, USA; ${ }^{4}$ Department of Malignant \\ Hematology, H. Lee Moffitt Cancer Center and Research Institute, Tampa, FL, USA
}

Myelodysplastic syndrome (MDS) is a diverse hematological malignancy with a wide spectrum of presentations and implications. Treatment strategies for patients with MDS heavily rely on prognostic scoring systems, such as the revised international prognostic scoring system (IPSS-R). Bone marrow fibrosis (BMF) has been identified as an independent risk factor for poor survival in patients with MDS, irrespective of the IPSS-R risk category. However, BMF is not widely included in scoring systems and is not always considered by clinicians when making treatment decisions for patients. In this review, we discuss the available literature about the presentation and prognosis of patients with MDS and concurrent BMF. The prognostic impact of BMF should be factored in when deciding on transplant candidacy, especially for intermediate-risk patients.

Key Words: Myelodysplastic syndrome, Bone marrow fibrosis, International prognostic scoring system, Allogeneic stem cell transplantation

\author{
Received: September 1, 2021 \\ Revision received: September 25, 2021 \\ Accepted: November 29, 2021
}

Corresponding author: Akriti G Jain, M.D. Department of Hematology and Oncology, H. Lee Moffitt Cancer Center and Research Institute, 12902 USF Magnolia Drive,

Tampa, FL, 33612, USA

Tel: +1-330-322-9730.

E-mail: Akriti.jain@moffitt.org

\section{cC) (i) (5)}

(c) Korean Society for Laboratory Medicine This is an Open Access article distributed under the terms of the Creative Commons Attribution Non-Commercial License (https://creativecommons.org/licenses/by-nc/4.0) which permits unrestricted non-commercial use, distribution, and reproduction in any medium, provided the original work is properly cited.

\section{INTRODUCTION}

The stromal microenvironment of the bone marrow (BM) comprises structural fibrils that provide connective tissue structure and support for hematopoietic progenitor cells. Reticulin and collagen type I/III are the most common BM fibers [1]. BM biopsy sections can be assessed for the presence of these stromal fibers to evaluate the extent of fibrosis using a silver impregnation technique called Gomori's stain [2]. A pathological increase in BM fibrosis (BMF) reportedly has both clinical and prognostic significance in various hematological malignancies. In chronic myeloid leukemia and multiple myeloma, BMF portends decreased responsiveness to most commonly used treatments [3, 4]. In primary myelofibrosis (PMF), the higher the fibrosis grade, the poorer the prognosis [5]. In patients with essential thrombocy- themia, increased reticulin fibrosis predicts an increased risk of thrombosis, major bleeding, and transformation into myelofibrosis, and in patients with polycythemia vera, it is associated with worse outcomes [6].

Myelodysplastic syndrome (MDS) is a heterogenous hematological malignancy with a wide spectrum of clinical presentations. MDS is characterized by dysplasia in one or more BM cell lineages and/or ineffective erythropoiesis. In 1981, Sultan, et al. [7] first described eight cases of MDS with BMF at presentation. The disease presentation described was later included in the category of acute myeloid leukemia (AML), not otherwise specified as acute panmyelosis with myelofibrosis [8]. In 1989, Pagliuca, et al. [9] described 10 cases of primary MDS with striking BMF at presentation. Since then, multiple case reports, case series, and retrospective studies have reported MDS with fibro- 
sis (MDS-F). MDS-F accounts for $10 \%-20 \%$ of MDS cases [1013].

The risk stratification scale of the revised international prognostic scoring system (IPSS-R) for MDS ranges from very low risk to very high risk [14]. The IPSS-R utilizes cytogenetics, BM blasts, and laboratory parameters, including $\mathrm{Hb}$, platelets, and absolute neutrophil count (ANC), at diagnosis [14]. BMF is not included in the IPSS-R for determining the risk category and prognosis. In addition, the clinical relevance of BMF was not recognized in the 2008 WHO classification [15]. The 2016 WHO classification identified MDS-F as an unclassified MDS subtype [8]. Moderate to severe BMF in de novo MDS is closely associated with multilineage dysplasia, transfusion dependence, and severe cytopenia [11, 16, 17]. Overall survival (OS) is inferior in de novo MDS patients with moderate to severe BMF. Poorer OS has been attributed to greater BM failure or a higher rate of leukemic transformation [10]. In patients with MDS who proceed to a hematopoietic allogeneic stem cell transplant (alloSCT), moderate to severe BMF is an independent risk factor for an inferior event-free survival. Delayed engraftment post alloSCT has been reported in MDS patients with any degree of BMF [18]. BMF can be seen in the initial diagnostic marrow or sometimes can develop during the course of MDS or after treatment. In a study by Fu, et al. [12], out of 551 patients with no significant BMF at diagnosis, 17 (4\%) patients developed BMF during follow-up, and the median time to development of BMF was 22 months.

While the pathophysiology of BMF is not well understood, some theories suggest increased cytokine production from megakaryocytes and platelets, which reportedly are higher in patients with BMF [19]. Cytokine transforming growth factor- $\beta$ has been suggested to be a potent stimulator of fibroblast collagen synthesis [19]. Other cytokines implicated in the pathophysiology of BMF include platelet-derived growth factor, calmodulin, and basic fibroblast growth factor [20]. Spliceosome and RAS pathway mutations have been implicated in the pathogenesis of BMF in patients with MDS [21]. However, it is clear that the BMF seen in MDS is a reactive process and that the fibroblasts present in the BM responsible for the fibrosis are not monoclonal [19]. We describe patients with MDS with BMF and their diagnosis, management, and prognosis.

\section{Grading of BMF}

Before 2005, the BMF grading was subjective, and pathologists used various grading systems [1]. In 2001, Steensma, et al. [20] attempted to define MDS-F. They used the Manoharan scale and recommended that only patients with grade $3+$ or $4+$ be di- agnosed as having MDS-F [22]. In 2005, the European Myelofibrosis Network framed a consensus-based proposal for semiquantitative evaluation of BMF to achieve a higher level of reproducibility in clinical practice [23]. Four categories were introduced to simplify the grading of marrow fibrosis (MF). MF-0 or no fibrosis in normal BM is used for patients with scattered linear reticulin without any intersections (cross-overs). Mild fibrosis (MF-1) is defined as a loose network of reticulin fibers with numerous intersections, particularly in the perivascular areas. Moderate fibrosis (MF-2) is graded when diffuse and dense reticulin with extensive intersections is noted, with focal and occasional bundles of collagen and/or osteosclerosis. In severe or grade 3 fibrosis (MF-3), there can be coarse bundles of collagen (on trichrome stain), frequently accompanying significant osteosclerosis in addition to diffuse and dense reticulin with extensive intersections [17]. MF-0/1 is present in up to $50 \%$ of MDS cases [7, $9,13]$. In this review, we discuss the significance of moderate to severe fibrosis (MF-2/3).

\section{Phenotype of MDS-F}

There are two types of fibers that contribute to BMF. While an increase in reticulin fibers has a limited association with the severity of the underlying malignancy, collagen fibers are strongly linked with abnormal blood counts and poor outcomes [1]. Reticulin fibrosis is frequently reversed after therapeutic intervention, while collagen fibrosis is less likely to alleviate with therapy [1]. Trichrome stain for collagen fibrosis is not commonly used for MDS-F as it is often, though not always, correlated with reticulin fibrosis ( $\geq$ MF-2). Fig. 1 shows peripheral blood and BM findings in a patient with MDS-F. BMF is often associated with dry tap which could hinder morphologic assessment of dysplasia. Careful examination of peripheral blood and the slides from bone marrow touch imprint would be helpful.

In a study on the clinical significance of moderate to severe BMF in patients with therapy-related (t-)MDS, Fu, et al. [24] found that 47 out of $266 \mathrm{t}$-MDS patients (17\%) had moderate to severe BMF. Clinically, patients with moderate to severe BMF had more severe thrombocytopenia $\left(42 \times 10^{9} / \mathrm{L}\right.$ vs. $62 \times 10^{9} / \mathrm{L}$, $P=0.039)$ and tended to have more circulating blasts $(P=0.051)$ than patients with mild BMF, whereas the rates of other clinical symptoms, including anemia, neutropenia, hepatosplenomegaly, transfusion requirements, and constitutional symptoms, were alike. Risk factors for BMF were prior solid tumor (46.8\% vs. $28.3 \%$ in prior hematological malignancies) and radiation therapy. Fu, et al. [12] found substantial BMF (MF-2/3) in 79 (13\%) out of 630 MDS patients. On comparing patients with substan- 
Jain AG, et al.

MDS with bone marrow fibrosis
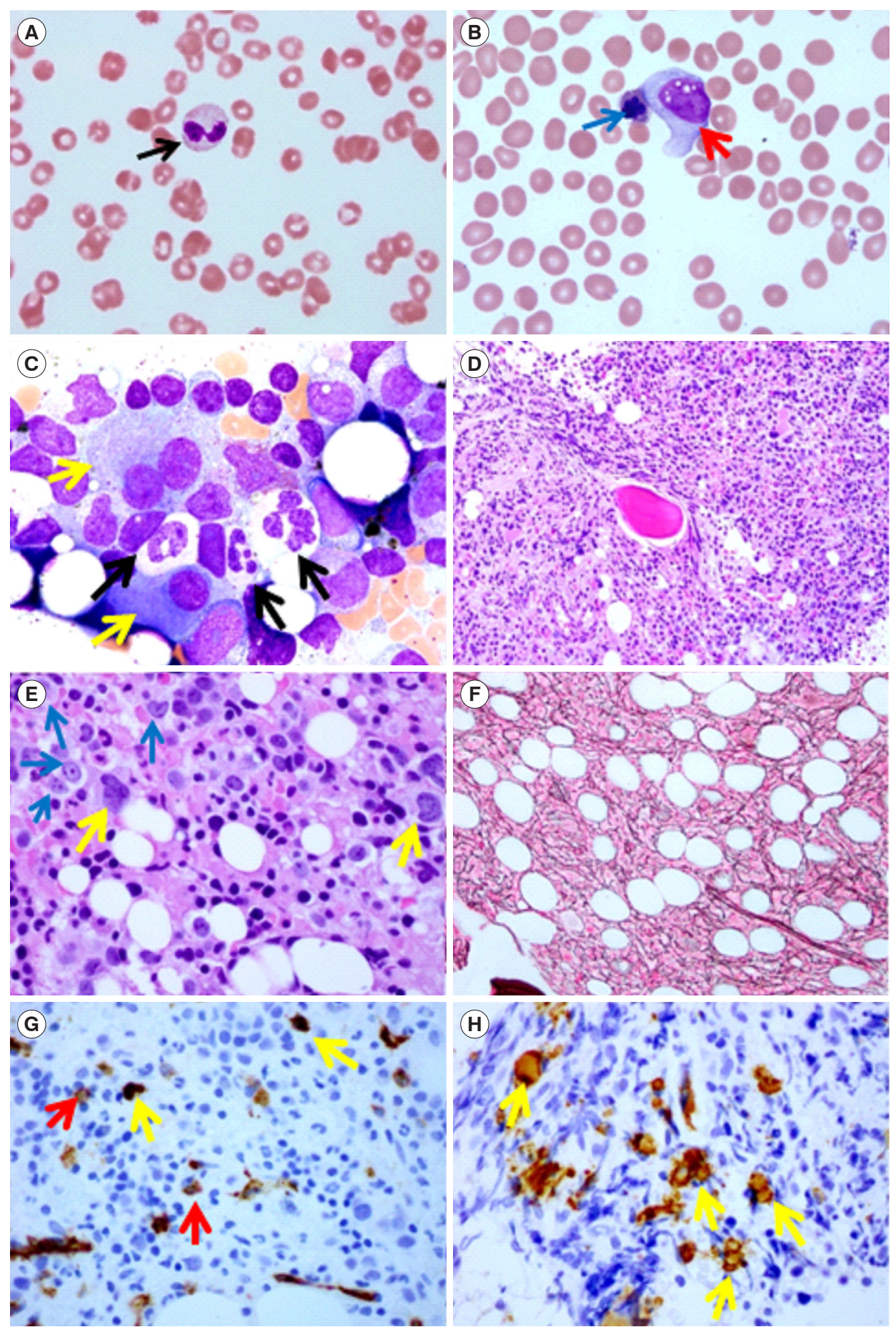

Fig. 1. Fibrotic MDS. (A, B) Peripheral blood smears revealing dysplastic neutrophils with pseudo-Pelger-Huet changes (black arrow-bilobed neutrophil, usually indicates an underlying BM disorder) and circulating dysplastic erythroid precursors with multilobulated nuclei (blue arrow) and a circulating blast (red arrow) - leukoerythroblastosis (Wright stain, $\times 1,000$ ). (C) BM touch imprint smear showing hypogranulated neutrophils (black arrows) and a bilobed megakaryocyte (yellow arrow) (Wright-Giemsa stain, $\times 1,000)$. (D, E) BM core biopsy showing hypercellularity (>90\%) associated with increased left-shifted erythroid precursors (blue arrow), dysplastic megakaryocytes (yellow arrow), decreased myeloid precursor numbers, and patchy crush artifact (hematoxylin and eosin stain, $\times 200$ [D], and $\times 600$ [E]). (F) Reticulin stain ( $\times 200$ ) showing moderate reticulin fibrosis (MF-2/3). (G) CD34-stained (brown colored) occasional myeloblasts (red arrows) and occasional small megakaryocytes/megakaryoblasts (yellow arrows) and (H) CD61-stained megakaryocytes, including small forms (immunoperoxidase stain, $\times 200$ ).

Abbreviation: MDS, myelodysplastic syndrome; BM, bone marrow. 
tial BMF to patients that did not have MF-2/3 fibrosis, they found that hepatosplenomegaly, constitutional symptoms, and transfusion dependence were more frequently observed in patients with substantial BMF. They also noted that although white blood cell counts, ANCs, and $\mathrm{Hb}$ levels were similar between the two groups, thrombocytopenia was more severe in patients with substantial BMF (51 vs. $94 \times 10^{9} / \mathrm{L}$ ) [12]. BM dysplasia was similar between patients without or with minimal BMF and patients with substantial BMF. As expected, patients with substantial BMF had higher megakaryocyte numbers and lower cellularity. However, they did not note a difference in the number of BM blasts [12].

In a study conducted at our institution by Melody, et al. [25], using our MDS database of 2,624 patients, MF-3 BMF was observed in only $4 \%$ of patients. Since preliminary analyses had indicated that only MF-3 affected survival, patients with MF-3 were compared with those with MF-0-2 fibrosis. In line with previous findings [12, 24], we noted that thrombocytopenia and a higher BM blast percentage were common in patients with MF-3 BMF. Interestingly, lactate dehydrogenase levels were higher in MF-3 patients ( $46 \%$ vs. $34 \%, P=0.02$ ).

Ramos, et al. [26] reported that BMF is positively correlated with ferritin and erythropoietin levels, marrow cellularity, abnormal localization of immature precursors (ALIP), packed red blood concentrate transfusions in the first 16 weeks from diagnosis, p53 immunohistochemistry scores, and peripheral Wilm's tumor 1 gene expression. Marisavljević, et al. [27] studied 236 patients with de novo MDS and found that immature cells and blasts in the peripheral blood and BM blasts were more frequently present in patients with MDS-F. In the BM, ALIP, dysmegakaryopoesis, and dyserythropoesis were also significantly higher in patients with MDS-F.

\section{Genotype of primary MDS with fibrosis}

It is imperative to recognize cytogenetic and mutational profiles that can help in BMF prognosis assessment. Cytogenetic features of MDS-F include monosomy 7 [9, 16, 28]. Complex or other unique cytogenetic features have been reported [7, 9, 16, 28-32]. Fu, et al. [24] studied 266 t-MDS patients and reported that monosomy 5 and monosomy 17 were more commonly detected in t-MDS patients with MF-2/3 $(P=0.031$ and $P=0.043$, respectively). In another study including 79 patients with substantial BMF, they noted that the distribution of cytogenetic risk using the new comprehensive cytogenetic scoring system for primary MDS/oligoblastic AML was similar [12]. In contrast to the findings by Fu, et al. [24], we noted poor cytogenetic and IPSS-R risk in patients with MF-3 BMF, but complex karyotypes were more commonly seen in these patients (31\% vs. $16 \%, P=$ 0.002) than in patients with MF-1/2 [25].

$J A K 2, C A L R$, or MPL variants leading to the constitutional activation of the JAK-STAT pathway are conspicuous features of myeloproliferative neoplasms. Molecular changes observed in patients with fibrotic MDS may be similar to those observed in patients with myelofibrosis. However, mutations in JAK2, CALR, and $M P L$ are rare in MDS. Hussein, et al. [33] studied fibrosisrelated gene transcripts in 119 patients with MDS, 70 of whom had accompanying BMF. They found that BMPR2, SMAD3, and SMAD4 were expressed in both PMF and MDS-F. Although mutational profiles were not available for all patients in the study by Fu, et al. [24], they noted that the JAK2, V617F variant was more frequently detected in patients with BMF ( $21 \%$ vs. $2 \%$; $P=0.014)$ than in patients without BMF. The median allele burden for the JAK2 V617F variant was 22.5\% (5.5\%-46\%). Melody, et al. [25] reported that TP53 and SETBP1 variants are more frequently detected in patients with MF-3. Wang, et al. [21] recently studied 239 patients with MDS or MDS-AML with BMF and reported that U2AF1 variants were more common in patients with MF-2/3 ( $P=0.029)$. The inability of ruxolitinib to reduce $B M F$ in patients with myelofibrosis contends against a pathogenic role of the JAK-STAT pathway in fibrosis. Hence, the pathogenic mechanisms of BMF in MDS remain to be fully unraveled.

\section{Management of patients with fibrotic MDS}

Patients with MDS with substantial BMF are treated using similar strategies as those without fibrosis. Supportive care, immunomodulatory agents, hypomethylating agents, induction chemotherapy, and/or alloSCT can be used. Fu, et al. [12] reported that treatment with hypomethylating agents did not decrease BMF even in patients who attained complete hematological responses, whereas patients who underwent alloSCT showed a complete resolution of BMF.

AlloSCT is the only potentially curative treatment for MDS. However, alloSCT is associated with a high mortality rate and hence is generally offered only to patients with high- and very-high-risk MDS. As noted before, BMF is not considered when IPSS-R is used to score the prognostic risk, but if BMF accompanies rapidly progressing MDS, alloSCT should be considered. Table 1 lists studies that comparatively investigated the outcomes of patients with MDS-F that underwent alloSCT and MDS patients without BMF. In a study in 721 MDS patients who underwent alloSCT, Kroger, et al. [18] compared patients with MF-3 BMF $(\mathrm{N}=39)$ with those with MF-1/2 $(\mathrm{N}=199)$ and MF-0 $(\mathrm{N}=483)$ 
Table 1. Studies in patients with MDS with BMF undergoing BM transplantation

\begin{tabular}{|c|c|c|c|c|c|c|}
\hline Reference & Patients (N) & $\begin{array}{l}\text { Patients with fibrosis, } \\
\text { N (\%) }\end{array}$ & OS & AML transformation & 3-yr RFS & Engraftment (day) \\
\hline Wang, et al. 2021 [21] & $\begin{array}{c}239 \\
\text { (MDS + MDS-AML) }\end{array}$ & $\begin{array}{c}\text { MF-1 } 81 \text { (33.9) } \\
\text { MF-2/3 } 37 \text { (15.5) }\end{array}$ & $\begin{array}{c}3 \text {-yr 0S rate } 72 \% \text { vs. } \\
67.5 \% \text { vs. } 41.3 \% \\
(P=0.018)\end{array}$ & $\begin{array}{c}\text { MF- } 2 / 3 \text { vs. MF- } 1 \text { vs. } \\
\text { MF- } 0 \\
8.8 \% \text { vs. } 39.9 \% \text { vs. } \\
50 \%, P=0.028\end{array}$ & $\begin{array}{c}72.8 \% \text { vs. } 68.8 \% \text { vs. } \\
44.8 \% ; P=0.018\end{array}$ & $\begin{array}{c}\text { Neutrophils, } 13 \text { vs. } 13 \\
\text { vs. } 14 ; P=0.031 \\
\text { Platelets, } 17 \text { vs. } 17.5 \\
\text { vs. } 20.5 ; P=0.05\end{array}$ \\
\hline Scott, et al. 2007 [34] & 471 & 113 & HR, $1.21 ; P=0.16$ & & & $\begin{array}{l}\text { Platelets, } 17 \text { vs. } 28 ; \\
\qquad P<0.0001\end{array}$ \\
\hline Kroger, et al. 2011 [18] & 721 & $\begin{array}{c}\text { MF-1/2 } 199 \\
\text { MF-3 } 39\end{array}$ & $\begin{array}{l}\text { HR, } 1.13 \text { vs. } 1.94 \\
\quad P=0.002\end{array}$ & & $\begin{array}{l}\text { HR } 1.13 \text { vs. } 1.88 \\
\quad(P=0.003)\end{array}$ & $\begin{array}{c}\text { Platelets, } 16 \text { vs. } 17 \text { vs } \\
20 ; P=0.002\end{array}$ \\
\hline
\end{tabular}

Abbreviations: BM, bone marrow; BMF, bone marrow fibrosis; OS, overall survival; AML, acute myeloid leukemia; RFS, relapse free survival; MDS, myelodysplastic syndrome; MF-1/2/3, BMF grade 1/2/3: HR, hazard ratio.

Table 2. Studies comparing MDS patients with BMF with those without BMF

\begin{tabular}{|c|c|c|c|c|c|}
\hline Reference & Patients (N) & $\begin{array}{c}\text { Patients with fibrosis, } \\
\text { N (\%) }\end{array}$ & OS (months) & AML transformation (\%) & DFS \\
\hline Wang, et al. 2020 [35] & 157 & $\begin{array}{l}34(21.7) \\
24 \mathrm{MF}-1 \\
10 \mathrm{MF}-2\end{array}$ & 17.7 vs. $47.6 ; P=0.001$ & 20.3 vs. $41 ; P=0.013$ & $\begin{array}{c}13.5 \text { vs. } 42.0 \text { months, } \\
P=0.002\end{array}$ \\
\hline Fu, et al. 2014 [12] & 630 & $\begin{array}{l}\text { MF-2/3 } 79 \text { (13) } \\
\text { Control } 166\end{array}$ & 21 vs. $42 ; P=0.000$ & LFS 52 vs. $120 ; P=0.003$ & \\
\hline Della Porta, et al. 2009 [10] & 298 & $\begin{array}{c}\text { MF-1 } 128 \text { (43) } \\
\text { MF-2 } 45 \text { (15) } \\
\text { MF-3 } 7 \text { (2) }\end{array}$ & $\begin{array}{l}\text { Inferior in } 2 / 3 \text { vs. } 0 / 1 \\
\qquad P<0.0001\end{array}$ & & $\begin{array}{l}\text { Inferior in } 2 / 3 \text { vs. } 0 / 1 ; \\
\qquad P<0.0001\end{array}$ \\
\hline Maschek, et al. 1992 [32] & 352 & $61(17.3)$ & 10 vs. 28.9 & 36.6 in patients with BMF & \\
\hline Marisavljević, et al. 2004 [27] & 236 & $126(53.4)$ & 13 vs. $35 ; P=0.00055$ & 24.1 vs. 18.9 & \\
\hline Melody, et al. 2020 [25] & 2,624 & $\begin{array}{c}\text { MF-0-2 2,517 } \\
\text { MF-3 } 107\end{array}$ & 19 vs. $56 ; P=0.02$ (alloSCT) & 29 vs. $28 ; P=0.84$ & \\
\hline
\end{tabular}

Abbreviations: MDS, myelodysplastic syndrome; BMF, bone marrow fibrosis; OS, overall survival; AML, acute myeloid leukemia; DFS, disease-free survival; MF-1/2/3, BMF grade 1/2/3; LFS, leukemia-free survival; MF, myelofibrosis; alloSCT, allogeneic stem cell transplantation.

BMF. They found that neutrophil engraftment occurred later in patients with MF-1,2/3 than in patients with MF-0 (16 vs. 20 vs. 17 days, $P=0.002$ ). Scott, et al. [34] studied 471 patients undergoing alloSCT, including 113 patients with either MDS or AML arising from MDS. They also noted delayed engraftment in patients with BMF (hazard ratio [HR] 0.4; $P<0.001$ ). However, they did not find differences in OS, relapse-free survival (RFS), and non-relapse mortality (NRM) between patients with BMF and those without, in their cohort. On subgroup analysis of patients with intermediate or high disease risk according to the IPSS-R, they found that OS, RFS, and NRM were inferior in patients with BMF.

Wang, et al. [21], in their recent study in 239 MDS and secondary AML patients who underwent alloSCT, reported that OS and disease-free survival were significantly lower in patients with MF-2/3 than in patients with MF-0/1 $(P=0.018)$. BMF was an independent predictor for survival in patients with $\geq 10 \% \mathrm{BM}$ blasts at diagnosis, and these patients had improved outcomes if they achieved complete remission before alloSCT.

\section{Prognostic value of BMF}

The most important implication of BMF in MDS patients is its impact on their prognosis in terms of survival as well as progression. Table 2 compares outcomes of patients with MDS- $F$ with those of patients with MDS. Fu, et al. [24] in their study in 266 t-MDS patients, $17 \%$ of whom had MF-2/3 BMF, reported a similar risk of AML transformation (9/39 vs. 48/191, $P=0.482$ ) and a comparable OS (8 vs. 9 months, $P=0.926$ ) between patients with MF-2/3 BMF and those with MF-1 at the median follow-up of 11.5 months in both univariate and multivariate analyses. In their study in 79 patients with MF-2/3 BMF, Fu, et al. [12] noted that the rate of $A M L$ transformation was higher in patients with 
substantial BMF ( $37 \%$ vs. $26 \% ; P=0.057$ ), and OS ( 21 vs. 42 months; $P=0.000$ ) and leukemia-free survival (LFS; 52 vs. 120 months; $P=0.0003$ ) were inferior in patients with $\mathrm{BMF}$ than in patients who did not undergo alloSCT. Cox regression analysis revealed that BMF was an independent risk factor for inferior OS and LFS. In their study in 236 de novo MDS patients, Marisavljević, et al. [27] reported that MDS patients with BMF had a significantly inferior OS compared to those without BMF (13 vs. 35 months; $P=0.0005$ ), and the average time to AML transformation was significantly shorter in patients with BMF (32 vs. $>56$ months, $P=0.015$ )

Melody, et al. [25] found that OS was significantly shorter in patients with MF-3 BMF than in those with MF-0-2 BMF (17 vs. 39.4, 36.6, and 24.5 months, respectively, $P<0.005$ ). OS was shorter in all IPSS-R risk groups (low, intermediate, and high) when patients with MF-3 BMF were compared with those with MF-0-2 BMF. Multivariate analysis revealed that the HR for death was $1.6(95 \% \mathrm{Cl}, 1.2-1.9)(P<0.005)$ for MF-3 BMF compared with MF-0 BMF. Although there was no increase in the rate of AML transformation in patients with MF-3 BMF in our patient cohort ( $29 \%$ vs. $28 \%, P=0.84$ ), the median time to AML transformation was shorter in patients with MF-3 than in those with MF-1/2 (23 vs. 35 months, $P=0.001$ ) [25].

Fu, et al. [12] noted that patients who developed BMF during the course of MDS showed evidence of disease progression on BMF development. The median OS for these patients from the time of BMF development was only nine months. For MDS patients with BMF that go on to receive alloSCT, the 3-year relapse rate was higher in patients with BMF than in those without (47\% vs. $28 \%, P=0.04$ ) [34]. Accordingly, Wang, et al. [35] recently reported that mild to moderate BMF is predictive of a poor clinical outcome.

\section{CONCLUSION}

When the IPSS-R prognostic criteria were first established, BMF was considered a potential factor for determining the prognostic risk. However, it was discounted as an additive factor for predicting survival in MDS because of its low prevalence and discrepancies in the evaluation of the degree of BMF across institutions. We presented evidence that BMF is a poor prognostic variable, even if it is observed during the course of disease. It is recommended that BMF be integrated into currently used risk classification/stratification systems. The presence of moderate to severe BMF in patients with MDS should be accounted for when considering transplantation as a potential treatment strat- egy in patients with intermediate-risk disease. Future directions include further improving our understanding of the underlying pathobiology of BMF and assessing the impacts of treatments in patients with MDS-F.

\section{ACKNOWLEDGEMENTS}

None.

\section{AUTHOR CONTRIBUTIONS}

Jain AG wrote the manuscript. Zhang $L$ reviewed the manuscript and contributed to the images for the manuscript. Bennet $\mathrm{JM}$ and Komrokii R critically reviewed and corrected the manuscript.

\section{CONFLICTS OF INTEREST}

Jain AG, Zhang L, and Bennett JM do not have any conflicts of interest to report. Komroji $\mathrm{R}$ does not have any conflicts significant for this review. Komroji R: BMSCelgene: Consultancy, Membership on an entity's Board of Directors or Advisory Committees and Speakers Bureau, Jazz: Consultancy and Speakers Bureau, Novartis: Consultancy, Honoraria and Membership on an entity's Board of Directors or Advisory Committees, AbbVie: Consultancy, Acceleron: Consultancy, PharmaEssentia: Membership on an entity's Board of Directors or Advisory Committees, Taiho Oncology: Membership on an entity's Board of Directors or Advisory Committees, Geron: Consultancy.

\section{RESEARCH FUNDING}

None declared.

\section{ORCID}

Akriti G Jain

https://orcid.org/0000-0002-7238-9241

Ling Zhang https://orcid.org/0000-0002-1463-1458

John M Bennett https://orcid.org/0000-0001-7517-5567

Rami Komrokji

\section{REFERENCES}

1. Kuter DJ, Bain B, Mufti G, Bagg A, Hasserjian RP. Bone marrow fibrosis: pathophysiology and clinical significance of increased bone marrow stromal fibres. Br J Haematol 2007; 139:351-62.

2. Gömöri G. Silver impregnation of reticulum in paraffin sections. Am J 
Pathol 1937;13:993-1002.5.

3. Kvasnicka HM, Thiele J, Schmitt-Graeff A, Diehl V, Zankovich R, Niederle $\mathrm{N}$, et al. Bone marrow features improve prognostic efficiency in multivariate risk classification of chronic-phase $\mathrm{Ph} 1+$ chronic myelogenous leukemia: a multicenter trial. J Clin Oncol 2001;19:2994-3009.

4. Subramanian R, Basu D, Dutta TK. Significance of bone marrow fibrosis in multiple myeloma. Pathology 2007;39:512-5.

5. Vener C, Fracchiolla NS, Gianelli U, Calori R, Radaelli F, lurlo A, et al. Prognostic implications of the European consensus for grading of bone marrow fibrosis in chronic idiopathic myelofibrosis. Blood 2008;111: 1862-5.

6. Campbell PJ, Bareford D, Erber WN, Wilkins BS, Wright P, Buck G, et al. Reticulin accumulation in essential thrombocythemia: prognostic significance and relationship to therapy. J Clin Oncol 2009;27:2991-9.

7. Sultan C, Sigaux F, Imbert M, Reyes F. Acute myelodysplasia with myelofibrosis: a report of eight cases. Br J Haematol 1981;49:11-6.

8. Arber DA, Orazi A, Hasserjian R, Thiele J, Borowitz MJ, Le Beau MM, et al. The 2016 revision to the World Health Organization classification of myeloid neoplasms and acute leukemia. Blood 2016;127:2391-405.

9. Pagliuca A, Layton DM, Manoharan A, Gordon S, Green PJ, Mufti GJ. Myelofibrosis in primary myelodysplastic syndromes: a clinico-morphological study of 10 cases. Br J Haematol 1989;71:499-504.

10. Della Porta MG, Malcovati L, Boveri E, Travaglino E, Pietra D, Pascutto C, et al. Clinical relevance of bone marrow fibrosis and CD34-positive cell clusters in primary myelodysplastic syndromes. J Clin Oncol 2009;27: 754-62

11. Buesche G, Teoman H, Wilczak W, Ganser A, Hecker H, Wilkens L, et al. Marrow fibrosis predicts early fatal marrow failure in patients with myelodysplastic syndromes. Leukemia 2008;22:313-22.

12. Fu B, Jaso JM, Sargent RL, Goswami M, Verstovsek S, Medeiros LJ, et al. Bone marrow fibrosis in patients with primary myelodysplastic syndromes has prognostic value using current therapies and new risk stratification systems. Mod Pathol 2014;27:681-9.

13. Lambertenghi-Deliliers G, Orazi A, Luksch R, Annaloro C, Soligo D. Myelodysplastic syndrome with increased marrow fibrosis: a distinct clinico-pathological entity. Br J Haematol 1991;78:161-6.

14. Greenberg PL, Tuechler H, Schanz J, Sanz G, Garcia-Manero G, Solé F, et al. Revised international prognostic scoring system for myelodysplastic syndromes. Blood 2012;120:2454-65.

15. Vardiman JW, Thiele J, Arber DA, Brunning RD, Borowitz MJ, Porwit A, et al. The 2008 revision of the World Health Organization (WHO) classification of myeloid neoplasms and acute leukemia: rationale and important changes. Blood 2009;114:937-51.

16. Verhoef GE, De Wolf-Peeters C, Ferrant A, Deprez S, Meeus P, Stul M, et al. Myelodysplastic syndromes with bone marrow fibrosis: a myelodysplastic disorder with proliferative features. Ann Hematol 1991;63: 235-41.

17. Della Porta MG and Malcovati L. Myelodysplastic syndromes with bone marrow fibrosis. Haematologica 2011;96:180-3.

18. Kröger N, Zabelina T, van Biezen A, Brand R, Niederwieser D, Martino R, et al. Allogeneic stem cell transplantation for myelodysplastic syndromes with bone marrow fibrosis. Haematologica 2011;96:291-7.
19. Tefferi A. Pathogenesis of myelofibrosis with myeloid metaplasia. J Clin Oncol 2005;23:8520-30.

20. Steensma DP, Hanson CA, Letendre L, Tefferi A. Myelodysplasia with fibrosis: a distinct entity? Leuk Res 2001;25:829-38.

21. Wang J, Wang Q, Zhang H, He Y, Huang Y, Zhang R, et al. Moderate to severe marrow fibrosis as a more advanced risk factor for MDS and MDSAML patients with excess of blasts receiving allogeneic hematopoietic stem cell transplantation. Transplant Cell Ther 2021;27:666.e1-9.

22. Manoharan A, Horsley R, Pitney WR. The reticulin content of bone marrow in acute leukaemia in adults. Br J Haematol 1979;43:185-90.

23. Thiele J, Kvasnicka HM, Facchetti F, Franco V, van der Walt J, Orazi A. European consensus on grading bone marrow fibrosis and assessment of cellularity. Haematologica 2005;90:1128-32.

24. Fu B, OK CY, Goswami M, Xei W, Jaso JM, Muzzafar T, et al. The clinical importance of moderate/severe bone marrow fibrosis in patients with therapy-related myelodysplastic syndromes. Ann Hematol 2013;92:133543.

25. Melody M, Al Ali N, Zhang L, Ramadan H, Padron E, Sallman D, et al. Decoding bone marrow fibrosis in myelodysplastic syndromes. Clin Lymphoma Myeloma Leuk 2020;20:324-8.

26. Ramos F, Robledo C, Izquierdo-García FM, Suárez-Vilela D, Benito R, Fuertes $\mathrm{M}$, et al. Bone marrow fibrosis in myelodysplastic syndromes: a prospective evaluation including mutational analysis. Oncotarget 2016; 7:30492-503.

27. Marisavljević D, Rolović Z, Cemerikić V, Bosković D, Colović M. Myelofibrosis in primary myelodysplastic syndromes: clinical and biological significance. Med Oncol 2004;21:325-31.

28. Ohyashiki K, Sasao I, Ohyashiki JH, Murakami T, Iwabuchi A, Tauchi T, et al. Clinical and cytogenetic characteristics of myelodysplastic syndromes developing myelofibrosis. Cancer 1991;68:178-83.

29. Allen EF, Lunde JH, McNally R, Branda RE. A case of acute myelofibrosis with complex karyotypic changes: a type of myelodysplastic syndrome. Cancer Genet Cytogenet 1996;90:24-8.

30. Reilly JT and Dolan G. Proposed classification for the myelodysplasia/ myelofibrosis syndromes. Br J Haematol 1991;79:653.

31. Narayanan MN, Benaim ME, Geary CG, Harrison CJ. Hyperfibrotic myelodysplasia. Br J Haematol 1991;79:653-5.

32. Maschek H, Georgii A, Kaloutsi V, Werner M, Bandecar K, Kressel MG, et al. Myelofibrosis in primary myelodysplastic syndromes: a retrospective study of 352 patients. Eur J Haematol 1992;48:208-14.

33. Hussein K, Stucki-Koch A, Kreipe H. Profile of fibrosis-related gene transcripts and megakaryocytic changes in the bone marrow of myelodysplastic syndromes with fibrosis. Ann Hematol 2018;97:2099-106.

34. Scott BL, Storer BE, Greene JE, Hackman RC, Appelbaum FR, Deeg $\mathrm{HJ}$. Marrow fibrosis as a risk factor for posttransplantation outcome in patients with advanced myelodysplastic syndrome or acute myeloid leukemia with multilineage dysplasia. Biol Blood Marrow Transplant 2007; 13:345-54.

35. Wang N, Xu H, Li Q, Fang X, Liu J, Sui X, et al. Patients of myelodysplastic syndrome with mild/moderate myelofibrosis and a monosomal karyotype are independently associated with an adverse prognosis: longterm follow-up data. Cancer Manag Res 2020;12:5881-91. 